\title{
HARDY SPACES AND REARRANGEMENTS
}

\section{BY}

\author{
BURGESS DAVIS ${ }^{1}$
}

\begin{abstract}
ABSTRACr. Let $f$ be an integrable valued function on the unit circle in the complex plane, and let $g$ be the rearrangement of $f$ satisfying $g\left(e^{i \theta}\right)>g\left(e^{i \varphi}\right)$ if $0<\theta<\varphi<$ $2 \pi$.

Define

$$
G(\theta)=\int_{-\theta}^{\theta} g\left(e^{i \varphi}\right) d \varphi .
$$

It is shown that some rearrangement of $f$ is in $\operatorname{Re} H^{1}$, that is, the distribution of $f$ is the distribution of a function in $\operatorname{Re} H^{1}$, if and only if $\int_{0}^{\pi}|G(\theta) / \theta| d \theta<\infty$, and that, if any rearrangement of $f$ is in $\operatorname{Re} H^{1}$, then $g$ is. The existence and form of rearrangements minimizing the $H^{1}$ norm are investigated. It is proved that $f$ is in $\operatorname{Re} H^{1}$ if and only if some rotation of $f$ is in the space dyadic $H^{1}$ of martingales. These results are extended to other $H^{p}$ spaces.
\end{abstract}

1. Introduction. Let $T$ be the unit circle in the complex plane $\mathbf{C}$, let the measure $m$ on $T$ be given by $d m=d \theta / 2 \pi$, and if $h$ is a function on $T$ let $\|h\|_{p}=$ $\left(\int_{T}|h|^{p} d m\right)^{1 / p}$. Functions on $T$ which are equal a.e. $(m)$ are identified. Let $f$ be an integrable real valued function on $T$, denote the conjugate function of $f$ by $\tilde{f}$, and if $p>0$ define $\|f\|_{H^{p}}=\|f+i \tilde{f}\|_{p}$. The first part of this paper is concerned primarily with the space $\operatorname{Re} H^{1}$, which consists of those $f$ such that $\|f\|_{H^{1}}<\infty$. For a discussion of conjugate functions and Hardy spaces see [12], and for a survey of recent developments in this area and the spaces $\operatorname{Re} H^{p}$, see [6].

A real valued function $g$ on $T$ is said to have the same distribution as $f$, or to be a rearrangement of $f$, if $m\{f<\lambda\}=m\{g<\lambda\}$ for each real number $\lambda$, and in this case we write $f \approx g$. Let $f_{d}$ be the rearrangement of $f$ satisfying $f_{d}\left(e^{i \theta}\right)>f_{d}\left(e^{i \varphi}\right)$ if $0<\theta<\varphi<2 \pi$, and let $M_{f}(\theta)=M(\theta)=\int_{-\theta}^{\theta} f_{d}\left(e^{-i \varphi}\right) d \varphi$. The following theorem characterizes the distributions of functions in $\operatorname{Re} H^{1}$.

THEOREM 1.1. There is a rearrangement of $f$ in $\operatorname{Re} H^{1}$ if and only if $\int_{0}^{\pi}|M(\theta) / \theta| d \theta$ $<\infty$, and in this case $f_{d} \in \operatorname{Re} H^{1}$. There are absolute positive constants $C$ and $c$ such that

$$
C\|f\|_{H^{1}} \geqslant \int_{0}^{\pi}|M(\theta) / \theta| d \theta>c\left\|f_{d}\right\|_{H^{1}}-\|f\|_{1} .
$$

The quantity $\int_{0}^{\pi}|M(\theta) / \theta| d \theta$ depends only on the distribution of $f$, and is 0 if $-f \approx f$. A theorem of Zygmund [12, Vol. 1, p. 254] states that if $f>0$, then

Received by the editors September 4, 1979 and, in revised form, December 10, 1979.

AMS (MOS) subject classifications (1970). Primary 42A40, 42A36, 30A78, 30A40, 60G65, 60J65.

Key words and phrases. Hardy spaces, conjugate function, rearrangements, Brownian motion, martingale.

'Supported by an NSF Grant. 
$f \in \operatorname{Re} H^{1}$ if and only if $f \in L \log L$, i.e. $\int_{T}|f| \log [\max (1,|f|)] d m<\infty$. It is not hard to show directly that if $f \geqslant 0$, then $f \in L \log L$ if and only if $\int_{0}^{\pi}|M(\theta) / \theta| d \theta$ $<\infty$, which is also implied by the truth of Theorem 1.1 and Zygmund's result. Of course $L \log L$ is rearrangement invariant, while it is well known that $\operatorname{Re} H^{1}$ is not.

Similar results hold on the real line R. Denote the Hilbert transform of an integrable real valued function $h$ on $\mathbf{R}$ by $\tilde{h}$, let $h_{\delta}$ be the rearrangement of $h$ which is not positive and not increasing on $(-\infty, 0)$, and not negative and not increasing on $(0, \infty)$. Let $M(x)=\int_{-x}^{x} h_{\delta}(t) d t$. Then there are absolute positive constants $A$ and $a$ such that

$$
\begin{aligned}
A \int_{-\infty}^{\infty}|h(x)+i \tilde{h}(x)| d x & \geqslant \int_{0}^{\infty}|M(x) / x| d x \\
& \geqslant a \int_{-\infty}^{\infty}\left|h_{\delta}(x)+i \tilde{h_{\delta}}(x)\right| d x-\int_{-\infty}^{\infty}|h(x)| d x .
\end{aligned}
$$

The condition $\int_{0}^{\infty}|M(x) / x| d x<\infty$, interpreted as a condition on the distribution of a function, also characterizes the distributions of functions in $H^{1}\left(\mathbf{R}^{n}\right), n>2$. Versions of inequalities (1.1) and (1.2) are also proved for $H^{p}$ spaces for some other exponents $p$.

This paper is closely related to recent work of Albert Baernstein II in [2]. Let $f_{s}$ be the rearrangement of $f$ satisfying $f_{s}\left(e^{i \theta}\right)=f_{s}\left(e^{-i \theta}\right)$ and $f_{s}\left(e^{i \varphi}\right)<f_{s}\left(e^{i \theta}\right)$ if $0<\theta$ $<\varphi \leqslant \pi$. Baernstein proves that $f_{s}$ is an extremal rearrangement of $f$ with regard to a number of $H^{p}$ and conjugate $L^{p}$ norms for the exponents $0<p<2$, and Essen and Shea noticed that his arguments extend to the exponents $p>2$ as well. The full result is given by the series of equations

$$
\begin{aligned}
\left\|\tilde{f}_{s}\right\|_{p} \geqslant\|\tilde{f}\|_{p}, & 1<p<2, \\
\left\|\tilde{f}_{s}\right\|_{p} \leqslant\|\tilde{f}\|_{p}, & 2<p<\infty, \\
\left\|f_{s}\right\|_{H^{p}} \geqslant\|f\|_{H^{p}}, & 0<p<2, \\
\left\|f_{s}\right\|_{H^{p}} \leqslant\|f\|_{H^{p}}, & 2<p<\infty .
\end{aligned}
$$

The first or third of these inequalities for $p=1$ implies that all rearrangements of $f$ are in $\operatorname{Re} H^{1}$ if and only if $f_{s} \in \operatorname{Re} H^{1}$. Essen and Shea have recently shown, in [8], that $f_{s} \in \operatorname{Re} H^{1}$ if and only if $f \in L \log L$.

In $\$ 5$ examples are given to show that, if $0<p<1$, there are functions $f_{p}=f$ such that $\left\|\tilde{f}_{s}\right\|_{p}$ is smaller than $\|\tilde{f}\|_{p}$, answering a question raised in [3].

We investigate rearrangements of $f$ which are extremal in a manner opposite to the way $f_{s}$ is extremal. Let $R$ be the collection of all rearrangements of $f$. A rearrangement $h$ of $f$ will be called reverse extremal if

$$
\begin{array}{rlr}
\|\tilde{h}\|_{p} & =\inf _{g \in \Re}\|\tilde{g}\|_{p}, & 1<p<2, \\
\|\tilde{h}\|_{p} & =\sup _{\boldsymbol{g} \in \Re}\|\tilde{g}\|_{p}, & 2<p<\infty, \\
\|h\|_{H^{p}} & =\inf _{g \in \Re}\|g\|_{H^{p}}, & 0<p<2, \\
\|h\|_{H^{p}} & =\sup _{\boldsymbol{g} \in \Re}\|g\|_{H^{p}}, & 2<p<\infty .
\end{array}
$$


The following theorem is proved.

THEOREM 1.2. Suppose there are $n \geqslant 2$ distinct real numbers $a_{1}, a_{2}, \ldots, a_{n}$ such that $\sum_{i=1}^{n} m\left\{f=a_{i}\right\}=1$ and $m\left\{f=a_{i}\right\}>0$ for each $i$. Let $a=\sum_{i=1}^{n} a_{i} m\left\{f=a_{i}\right\}$. If none of the $a_{i}$ equals $a$, there is a reverse extremal rearrangement of $f$. If $a_{i}=a$ for some $i$, there is no rearrangement $h$ of $f$ such that any of (1.3), (1.4), (1.5), or (1.6) hold for any $p$ in the specified intervals.

Even when they exist, the reverse extremal rearrangements of Theorem 1.2 are chaotic if $n>2$, being the real part of the boundary values of a universal covering map of a multiply connected region. For other, less discrete, distributions of $f$, reverse extremal distributions exist which are quite regular. The arrangement $f_{d}$ is never, except in trivial cases, reverse extremal.

If $n$ is a nonnegative integer, and $k$ is an integer satisfying $1<k<2^{n}$, let $A(k, n)=\left\{e^{i \theta}: 2 \pi(k-1) 2^{-n}<\theta<2 \pi k 2^{-n}\right\}$ so that the $\operatorname{arcs} A(k, n)$ partition $T$ into $2^{n}$ arcs of equal length. Let $\mathscr{D}_{n}$ be the $n$th dyadic $\sigma$-field, the one generated by $\left\{A(k, n), 1 \leqslant k \leqslant 2^{n}\right\}$, and, if $f$ is a real valued integrable function on $T$, let $f_{n}=E\left(f \mid \mathscr{D}_{n}\right)$ be the function which is constant on each $A(k, n)$ and which satisfies

$$
\int_{A(k, n)} f d m=\int_{A(k, n)} f_{n} d m, \quad 1<k<2^{n} .
$$

The sequence $f_{0}, f_{1}, \ldots$ is a martingale, and $f$ is said to be in the space $H^{1}$ (dyadic) if $\int_{T} \sup _{n}\left|f_{n}\right| d m<\infty$. These spaces have been extensively studied (see [9]). It is known that $H^{1}$ (dyadic) is contained in $\operatorname{Re} H^{1}$ but that there are functions in $\operatorname{Re} H^{1}$ which are not in $H^{1}$ (dyadic). In $\S 3$ is shown that if $f$ is in $\operatorname{Re} H^{1}$ then $f\left(e^{i(\theta+\varphi)}\right)=g(\theta)$ is in $H^{1}$ (dyadic) for almost every $\varphi \in[0,2 \pi)$.

The proofs of the results described use probability theory. A probabilistic characterization of $H^{p}$ due to Burkholder, Gundy, and Silverstein is used in the proof of Theorem 1.1, and optimal stopping, together with ideas developed by Baernstein in [2], is used to prove Theorem 1.2.

The author benefited from discussions with John L. Lewis. While this paper was being refereed Lewis, Baernstein, and P. Jones found a nonprobabilistic proof of Theorem 1.1. Their proof of the left-hand inequality in (1.1) is based on duality and the new result of Coifman and Rochberg that if $f>0$ is integrable then the logarithm of the Hardy-Littlewood maximal function is $B M O$.

2. Distributions of $H^{p}$ functions. If $f$ is an integrable function on $T$ we define the analytic function $F$ in the unit disc $D$ by

$$
F(z)=\int_{T}\left[\left(e^{i \theta}+z\right) f\left(e^{i \theta}\right) /\left(e^{i \theta}-z\right)\right] d m, \quad z \in D,
$$

and extend $F$ to $T$ by defining $F\left(e^{i \theta}\right)=f\left(e^{i \theta}\right)+i \tilde{f}\left(e^{i \theta}\right)$. We write $F(z)=u^{f}(z)+$ $i v^{f}(z)=u(z)+i v(z),|z| \leqslant 1$, the superscript being omitted when the reference function is clear from context. Note that $f\left(e^{i \theta}\right)=u\left(e^{i \theta}\right)$ and $\tilde{f}\left(e^{i \theta}\right)=v\left(e^{i \theta}\right)$. The function $u(z)$ is defined to be 0 if $|z|>1$. In this section the inequalities (1.1) and (1.2) will be proved together with versions for some other $H^{p}$ norms. The symbols $c_{p}, k_{p}$, etc. are absolute positive constants depending only on $p$ and may stand for 
different numbers from line to line. Versions of the inequalities to be proved hold for exponents $p>1$, but are either trivial or easy consequences of the M. Riesz inequalities $\|\tilde{f}\|_{p} \leqslant C_{p}\|f\|_{p}, p>1$, [12, Vol. 1, p. 253], so usually only the exponents $0<p \leqslant 1$ are considered.

Let $f_{d}$ and $M$ be as in the introduction, let $b_{n}=\pi 2^{-n}$, and if $p>0$ define

$$
A_{p}(f)=\sum_{n=0}^{\infty} 2^{n(p-1)}\left|M\left(b_{n}\right)\right|^{p} .
$$

LEMMA 2.1. If $f$ is an integrable function on $T$ and $0<p<1$ then

$$
c_{p} A_{p}(f)-C_{p}\|f\|_{p}^{p} \leqslant \int_{0}^{\pi}|M(\theta) / \theta|^{p} d \theta<K_{p}\left(A_{p}(f)+\|f\|_{p}^{p}\right) .
$$

Proof. Let $h(\theta)=\left|f_{d}\left(e^{i \theta}\right)\right|+\left|f_{d}\left(e^{-i \theta}\right)\right|$. Now

$$
\begin{aligned}
\int_{b_{n+1}}^{b_{n}} \theta^{-p}|M(\theta)|^{p} d \theta \geqslant & \int_{b_{n+1}}^{b_{n}} b_{n}^{-p}|M(\theta)|^{p} d \theta \\
\geqslant & \int_{b_{n+1}}^{b_{n}} b_{n}^{-p}\left|M\left(b_{n}\right)\right|^{p} d \theta \\
& -\left.\int_{b_{n+1}}^{b_{n}} b_{n}^{-p} \max _{b_{n+1}<\varphi<b_{n}}|| M\left(b_{n}\right)\right|^{p}-|M(\varphi)|^{p} \mid d \theta \\
\geqslant & 2^{-1} b_{n}^{(1-p)} M\left(b_{n}\right)^{p}-\int_{b_{n+1}}^{b_{n}} b_{n}^{-p} \max _{b_{n+1}<\varphi<b_{n}}\left|M\left(b_{n}\right)-M(\varphi)\right|^{p} d \theta \\
\geqslant & 2^{-1} b_{n}^{(1-p)} M\left(b_{n}\right)^{p}-2 \int_{b_{n+1}}^{b_{n}} h\left(b_{n+1}\right)^{p} d \theta .
\end{aligned}
$$

Summing these inequalities for $n=0,1,2, \ldots$ we get

$$
\begin{aligned}
\int_{0}^{\pi} \theta^{-p}|M(\theta)|^{p} d \theta & \geqslant\left(2 \pi^{(p-1)}\right)^{-1} A_{p}(f)-2 \sum_{n=0}^{\infty} \int_{b_{n+1}}^{b_{n}} h\left(b_{n+1}\right)^{p} d \theta \\
& \geqslant\left(2 \pi^{(p-1)}\right)^{-1} A_{p}(f)-4 \sum_{n=0}^{\infty} \int_{b_{n+2}}^{b_{n+1}} h\left(b_{n+1}\right)^{p} d \theta \\
& \geqslant\left(2 \pi^{(p-1)}\right)^{-1} A_{p}(f)-4 \int_{-\pi}^{\pi}\left|f_{d}\left(e^{i \theta}\right)\right|^{p} d \theta \\
& =\left(2 \pi^{(p-1)}\right)^{-1} A_{p}(f)-4\|f\|_{p}^{p},
\end{aligned}
$$

proving the left-hand inequality of Lemma 2.1. The proof of the right-hand side is similar.

Now the left-hand side of (1.1) will be proved. An expository treatment of the probability theory used may be found in [7]. The proof is based on a probabilistic characterization of $H^{p}$ given by Burkholder, Gundy, and Silverstein in [4]. The process $Z_{t}=X_{t}+i Y_{t}, t>0$, will denote standard two dimensional Brownian motion, and $P_{z}$ and $E_{z}$ will stand for probability and expectation associated with $Z_{t}$ started at $z$, with $P_{0}$ and $E_{0}$ shortened to $P$ and $E$. If $R$ is a region in $C$, the stopping time $\tau_{R}$ is defined by $\tau_{R}=\inf \left\{t>0: Z_{t} \notin R\right\}$. If $g$ is a function on $\bar{D}$, the closure of $D$, and $Z_{0} \in \bar{D}$, we define $g^{*}=\sup _{0<t<\tau_{D}}\left|g\left(Z_{t}\right)\right|$. It is proved in [4] 
that

$$
c_{p} E\left(u^{*}\right)^{p}<\|f\|_{H^{p}}<C_{p} E\left(u^{*}\right)^{p}, \quad 0<p<\infty .
$$

If $r$ is a real number let $r_{+}=\max (r, 0)$ and $r_{-}=\max (-r, 0)$. Then (2.1) implies $f$ is in $H^{p}$ if and only if both $E\left(u_{+}^{*}\right)^{p}$ and $E\left(u_{-}^{*}\right)^{p}$ are finite.

Since $f$ is integrable, $\lim _{t \rightarrow \tau_{D}} u\left(Z_{t}\right)=u\left(Z_{\tau_{D}}\right)$, by a theorem of Doob (see [7]). Until further mention it will be assumed that $\int_{T} f d m=0$. Define, for each positive real number $\lambda$, the stopping time $t_{\lambda}$ by $t_{\lambda}=\inf \left\{t>0: u\left(Z_{t}\right)=\lambda\right\}$, and let $p_{\lambda}$ be the density with respect to $m$ of $Z_{\tau_{D}} I\left(t_{\lambda}<\tau_{D}\right)$, meaning that if $A$ is an arc of $T$,

$$
\int_{A} p_{\lambda}\left(e^{i \theta}\right) d m=P\left(Z_{\tau_{D}} \in A \text { and } t_{\lambda}<\tau_{D}\right) .
$$

Note $\left\{t_{\lambda}<\tau_{D}\right\}=\left\{u_{+}^{*}>\lambda\right\}$. Clearly,

$$
p_{\lambda}=1 \text { on }\{f>\lambda\} \text {, }
$$

while, since $Z_{\tau_{D}}$ is uniformly distributed under $P$ on $T$,

$$
0 \leqslant p_{\lambda} \leqslant 1 \text {. }
$$

Now $E_{z} f\left(Z_{\tau_{D}}\right)=u(z), z \in D$, so that, using the strong Markov property, and the fact that $u\left(Z_{t \wedge \tau_{D}}\right), t>0$, is a martingale, we have

$$
\begin{aligned}
\int_{T} f\left(e^{i \theta}\right) p_{\lambda}\left(e^{i \theta}\right) d m & =E f\left(Z_{\tau_{D}}\right) I\left(t_{\lambda}<\tau_{D}\right) \\
& =E E_{Z_{t_{\lambda}}} f\left(Z_{\tau_{D}}\right) I\left(t_{\lambda}<\tau_{D}\right)=E u\left(Z_{t_{\lambda}}\right) I\left(t_{\lambda}<\tau_{D}\right) \\
& =E \lambda I\left(t_{\lambda}<\tau_{D}\right)=\lambda P\left(t_{\lambda}<\tau_{D}\right) .
\end{aligned}
$$

Since $P\left(t_{\lambda}<\tau_{D}\right)=P\left(u_{+}^{*}>\lambda\right)=\int_{T} p_{\lambda}\left(e^{i \theta}\right) d m$, this implies

$$
\lambda \int_{T} p_{\lambda}\left(e^{i \theta}\right) d m=\int_{T} f\left(e^{i \theta}\right) p_{\lambda}\left(e^{i \theta}\right) d m .
$$

It is not hard to show that among all functions $h$ which satisfy (2.2), (2.3), and (2.4) with $h$ in place of $p_{\lambda}, \int_{T} h d m$ is minimized when $h=I(H)$, where $H$ is of the form $H=\{f>\lambda\} \cup\{f<\alpha\} \cup G$, where $G \subset\{f=\alpha\}$, and $\alpha$ and $G$ are chosen so that $I(H)$ satisfies (2.4) with $I(H)$ in place of $p_{\lambda}$.

Let $\theta_{\lambda}=\sup \left\{\theta>0: f_{d}\left(e^{i \theta}\right)>\lambda\right\}$. Note $\theta_{\lambda}<2 \pi$ since we are assuming $\int_{T} f d m$ $=0$. Let $\varphi_{\lambda}$ be the unique number in $(-2 \pi, 0]$ such that

$$
\int_{\varphi_{\lambda}}^{\theta_{\lambda}} f_{d}\left(e^{i \theta}\right) d m=\lambda\left(\theta_{\lambda}-\varphi_{\lambda}\right) / 2 \pi
$$

For positive $\lambda$ define $\psi_{\lambda}=\left(\theta_{\lambda}-\varphi_{\lambda}\right) / 2 \pi$, and take $\psi_{0}=1$. Then if $\lambda>0, \psi_{\lambda}=$ $m(H)$, where $H$ is the set described in the previous paragraph, which can be seen by observing that, if $f=f_{d}$, one choice for $H$ is $\left\{e^{i \theta}: \varphi_{\lambda}<\theta<\theta_{\lambda}\right\}$. Thus,

$$
\psi_{\lambda}=m(H)<\int_{T} p_{\lambda}\left(e^{i \theta}\right) d m=P\left(u_{+}^{*}>\lambda\right),
$$

so that, if $p>0$,

$$
E\left(u_{+}^{*}\right)^{p}=p \int_{0}^{\infty} \lambda^{p-1} P\left(u_{+}^{*} \geqslant \lambda\right) d m \geqslant p \int_{0}^{\infty} \lambda^{p-1} \psi_{\lambda} d \lambda .
$$


Now let

$$
\alpha_{n}=\left(2^{n} \int_{-b_{n}}^{b_{n}} f_{d}\left(e^{i \theta}\right) d m\right)_{+}
$$

If $\alpha_{n}>0, \psi_{\alpha_{n}} \geqslant 2^{-(n+1)}$. This is clear if $\theta_{\alpha_{n}}>b_{n}$, while when $0<\theta_{\alpha_{n}}<b_{n}, f_{d}\left(e^{i \theta}\right)<$ $\alpha_{n}$ if $\theta \in\left(\theta_{\alpha_{n}}, b_{n}\right)$ so that

$$
\begin{aligned}
\int_{-b_{n}}^{\dot{\theta}_{\alpha_{n}}} f_{d}\left(e^{i \theta}\right) d m & =\int_{-b_{n}}^{b_{n}} f_{d}\left(e^{i \theta}\right) d m-\int_{\theta_{\alpha_{n}}}^{b_{n}} f_{d}\left(e^{i \theta}\right) d m \\
& >2^{-n} \alpha_{n}-\alpha_{n}\left(b_{n}-\theta_{\alpha_{n}}\right)(2 \pi)^{-1} \\
& =\alpha_{n}\left(\theta_{\alpha_{n}}-\left(-b_{n}\right)\right) / 2 \pi,
\end{aligned}
$$

implying $\varphi_{\alpha_{n}} \leqslant-b_{n}$.

Now let $j_{1}=1$, let $j_{2}$ be the first $i>1$, if it exists, such that $\alpha_{i}>2 \alpha_{1}$, and in general, if $\alpha_{j_{i}}$ exists let $j_{i+1}$ be the first $k>j_{i}$, if it exists, such that $\alpha_{k}>2 \alpha_{j i}$. If $\left\{j_{i}\right.$ : $i \in A\}$ denotes the collection of integers obtained in this manner, for each integer $p>0$ we have

$$
\sum_{n=0}^{\infty} 2^{-(n+1)} \alpha_{n}^{p} \leqslant\left(1+2^{p}\right) \sum_{i \in A} 2^{-\left(i_{i}+1\right)} \alpha_{j_{i}}^{p} .
$$

This, and the fact that $\psi_{\lambda}$ is nonincreasing, give

$$
\begin{aligned}
E\left(u_{+}^{*}\right)^{p} & \geqslant p \int_{0}^{\infty} \psi_{\lambda} \lambda^{p-1} d \lambda \\
& \geqslant p \sum_{i \in A} \int_{\alpha_{j_{i} / 2}}^{\alpha_{j_{i}}} \psi_{\lambda} \lambda^{p-1} d \lambda \geqslant c_{p} \sum_{i \in A} \alpha_{j_{i}}^{p} 2^{-\left(j_{i}+1\right)} \\
& \geqslant c_{p} \sum_{n=0}^{\infty} \alpha_{n}^{p} 2^{-(n+1)}=c_{p} \sum_{n=0}^{\infty} 2^{n(p-1)}\left(\int_{-b_{n}}^{b_{n}} f_{d}\left(e^{i \theta}\right) d m\right)_{+}^{p} .
\end{aligned}
$$

Replacing $f$ by $-f$ in this inequality gives

$$
E\left(u_{-}^{*}\right)^{p}>c_{p} \sum_{n=0}^{\infty} 2^{n(p-1)}\left(\int_{-b_{n}}^{b_{n}} f_{d}\left(e^{i \theta}\right) d m\right)_{-}^{p} .
$$

The two inequalities above, together with (2.1), give

$$
\|f\|_{H^{p}}^{p^{p}} \geqslant c_{p} E\left(u^{*}\right)^{p}>c_{p} \max \left(E\left(u_{+}^{*}\right)^{p}, E\left(u_{-}^{*}\right)^{p}\right)>c_{p} A_{p}(f), \quad 0<p<\infty .
$$

This inequality was proved under the assumption that $\int_{T} f d m=0$. Now we drop this assumption and extend (2.6) to arbitrary integrable $f$. Let $e=\int_{T} f d m$, and let $g=f-e$. Then since $u^{g}(0)=0,2\left(u^{f}\right)^{*}>\left(u^{g}\right)^{*}$. Also

$$
A_{p}(f)<C_{p}\left(A_{p}(g)+|e|^{p}\right) \leqslant C_{p} A_{p}(g)+C_{p} E\left(\left(u^{f}\right)^{*}\right)^{p} .
$$

Since, by (2.6)

$$
A_{p}(g) \leqslant C_{p} E\left(\left(u^{g}\right)^{*}\right)^{p}
$$

we have

$$
A_{p}(f)<C_{p} A_{p}(g)+C_{p} E\left(\left(u^{f}\right)^{*}\right)^{p}<C_{p} E\left(\left(u^{f}\right)^{*}\right)^{p}<C_{p}\|f\|_{H^{p}}^{p}
$$


Together with Lemma 2.1 and the fact that $\|f\|_{H^{p}}>\|f\|_{p}$ this gives

THEOREM 2.1. If $f$ is an integrable function on $T$ then

$$
\int_{0}^{\pi}|M(\theta) / \theta|^{p} d \theta \leqslant C_{p}\|f\|_{H^{p}}^{p}, \quad 0<p \leqslant 1 .
$$

Now the right side of (1.1) will be proved and generalized. Let $n$ be a positive integer, and, if $k$ is an integer satisfying $1 \leqslant k \leqslant 2^{n}$, let $A(k, n)$ be the arc $\left\{e^{i \theta}\right.$ : $\left.2 \pi(k-1) / 2^{n}<\theta<2 \pi k / 2^{n}\right\}$. Define $B(n)=A(1, n) \cup A\left(2^{n}, n\right)$. Let $\mathscr{F}_{1}$ be the trivial $\sigma$-field $\{\phi, T\}$, and if $n>1$, let $\mathscr{F}_{n}$ be the $\sigma$-field generated by the collection of $2^{n}-1$ intervals consisting of $B(n)$ together with $A(k, n), 2<k<2^{n}-1$. None of the intervals generating $\mathscr{F}_{n+1}$ is less than one quarter of the length of the smallest interval in $\mathscr{F}_{n}$ containing it. This property makes $\mathscr{F}_{n}, n>1$, a so-called regular family of $\sigma$-fields, and it makes the martingales introduced regular martingales.

If $g$ is an integrable function on $T$ define

$$
g_{n}=E\left(g \mid \mathscr{F}_{n}\right), \quad g_{n}^{*}=\max _{1<k<n}\left|g_{k}\right|, \quad \text { and } \quad g_{\infty}^{*}=\sup _{n>1}\left|g_{n}\right|
$$

It is easily shown that $g=\lim _{n \rightarrow \infty} g_{k}$. The function $g$ is said to be in the space $H^{p}\left(\mathscr{F}_{n}, n \geqslant 1\right)$ if $g_{\infty}^{*} \in L^{p}$. It is well known that a theory exists for these spaces which has many parallels to classical $H^{p}$ theory. An account of this may be found in [9].

Let $A$ be an arc of $T$, and let $h$ be a function on $T$ satisfying $\{h \neq 0\} \subset A$, $\int_{T} h d m=0$, and $|h|<m(A)^{-1}$. Then $h$ is an atom, using the terminology of Coifman (see [6]), and

$$
\|h\|_{H^{p}}^{p} \leqslant C_{p}\|h\|_{p}^{p}, \quad 1 / 2<p<\infty .
$$

The following lemma is essentially known. For $p=1$ it could be proved with duality arguments. Its proof is similar to arguments employed by C. Herz in [10].

LEMMA 2.2. Let $g$ be an integrable function on $T$. Then

$$
\|g\|_{H^{p}}^{p} \leqslant C_{p} \int_{T}\left(g_{\infty}^{*}\right)^{p} d m, \quad 1 / 2<p<1 .
$$

Proof. Let $x_{p}=\left(E\left(g_{\infty}^{*}\right)^{p}\right)^{1 / p}$, and assume $x_{p}<\infty$. Let $\tau_{0}=1$, and if $k>1$ let $\tau_{k}=\inf \left\{i: m\left(g_{i+1}^{*}>2^{k} x_{p} \mid \mathscr{F}_{i}\right)>0\right\}, \tau_{k}=\infty$ if no such $i$ exists. Let $g_{\infty}=g$. Then $\left|g_{\tau_{k}}\right|<2^{k} x_{p}$, while $m\left(\tau_{k}<\infty\right)<4 m\left(g_{\infty}^{*}>2^{k} x_{p}\right)$, due to the regularity of $\mathscr{F}_{n}$, $n \geqslant 1$. Let $G_{i}=\left(g_{\tau_{i}}-g_{\tau_{i-1}}\right) I\left(\tau_{i-1}<\infty\right)$, where $g_{\tau_{i}}=g$ on $\left\{\tau_{i}=\infty\right\}$. Then

$$
g=\sum_{i=1}^{\infty} G_{i}+\int_{T} g d m
$$

We can write $G_{i}=\sum_{j \in B} G_{i} I\left(A_{j}\right)$, where the $A_{j}$ are disjoint arcs in $\mathscr{F}_{\tau_{i-1}}$ such that $\sum_{j \in B} m\left(A_{j}\right)=m\left\{\tau_{i-1}<\infty\right\}$. Since $E\left(G_{i} \mid \mathscr{F}_{\tau_{i-1}}\right)=0, \int_{A_{j}} G_{i} d m=0$ for any $j \in B$. Since $\left|G_{i}\right| \leqslant 2^{i+1} x_{p},(2.7)$ gives

$$
\left\|G_{i}\right\|_{H^{p}}^{p} \leqslant C_{p} 2^{p(i+1)} x_{p}^{p} m\left\{\tau_{i-1}<\infty\right\}
$$


so

$$
\begin{aligned}
\|g\|_{H^{p}}^{p} & \leqslant\left|\int_{T} g d m\right|^{p}+C_{p} \sum_{i=1}^{\infty} 2^{p(i+1)} x_{p}^{p} m\left(\tau_{i-1}<\infty\right) \\
& \leqslant\left|g_{1}\right|^{p}+C_{p} \sum_{i=1}^{\infty} 2^{p(i+1)} x_{p}^{p} 4 m\left(g_{\infty}^{*}>2^{i-1} x_{p}\right) \\
& \leqslant\left|g_{1}\right|^{p}+C_{p} \int\left(g_{\infty}^{*}\right)^{p} d m<\left(C_{p}+1\right) \int\left(g_{\infty}^{*}\right)^{p} d m
\end{aligned}
$$

proving the lemma.

Now on each of the $\operatorname{arcs} A(k, n), 2 \leqslant k \leqslant 2^{n-1}$,

$$
\begin{aligned}
E\left(f_{d} \mid \mathscr{F}_{n}\right) & \leqslant \max \left\{\left|f_{d}\left(e^{i \theta}\right)\right|: e^{i \theta} \in A(k, n)\right\} \\
& \leqslant \max \left\{\left|f_{d}\left(e^{i \theta / 2}\right)\right|,\left|f_{d}\left(e^{-i \theta / 2}\right)\right|\right\}=\beta\left(e^{i \theta}\right) .
\end{aligned}
$$

Since

$$
\begin{aligned}
\left(f_{d}\right)_{\infty}^{*} & \leqslant \beta\left(e^{i \theta}\right)+\sup _{n>1}\left|E\left(f_{d} \mid \mathscr{F}_{n}\right) I\left(B_{n}\right)\right| \\
& \leqslant \beta\left(e^{i \theta}\right)+\sum_{n=1}^{\infty}\left|E\left(f_{d} \mid \mathscr{F}_{n}\right) I\left(B_{n}\right)\right| \\
& =\beta\left(e^{i \theta}\right)+\sum_{n=1}^{\infty} 2^{n}\left|\int_{B_{n}} f_{d} d m\right| I\left(B_{n}\right),
\end{aligned}
$$

we have, since $1 / 2<p \leqslant 1$,

$$
\begin{aligned}
\int_{T}\left[\left(f_{d}\right)_{\infty}^{*}\right]^{p} d m & \leqslant \int_{T}\left|\beta\left(e^{i \theta}\right)\right|^{p} d m+A_{p}(f) \\
& \leqslant 4 \int_{T}\left|f_{d}\left(e^{i \theta}\right)\right|^{p} d m+A_{p}(f) .
\end{aligned}
$$

Together with Lemma 2.2 this gives

$$
\left\|f_{d}\right\|_{H^{p}}^{p} \leqslant C_{p}\left(\|f\|_{p}^{p}+A_{p}(f)\right), \quad 1 / 2<p<1,
$$

and this with Lemma 2.1 proves the following theorem.

THEOREM 2.2. Let $f$ be an integrable function on $T$. Then

$$
\left\|f_{d}\right\|_{H^{p}}^{p} \leqslant C_{p}\left(\|f\|_{p}^{p}+\int_{0}^{\pi}|M(\theta) / \theta|^{p} d \theta\right), \quad 1 / 2<p<1 .
$$

The analog of (2.8) does not hold if $0<p<1 / 2$, since, if the distribution of $f$ is given by $m\{f=1\}=m\{f=-1\}=\alpha, m\{f=0\}=1-2 \alpha$, then $\int_{0}^{\pi}|M(\theta) / \theta|^{p} d \theta$ $=0$, and it can be shown that $\left\|f_{d}\right\|_{H^{p}}^{p} /\|f\|_{p}^{p}$ approaches infinity as $\alpha$ decreases to 0 , for any $p \in(0,1 / 2)$. Even so, we conjecture that, for these exponents, inf $\|g\|_{H^{p}}^{p}<$ $C_{p}\left(\|f\|_{p}^{p}+A_{p}(f)\right)$, where the infimum is taken over all rearrangements of $f$. In any event, the inequalities we have proved do not apply immediately to all the functions in $H^{p}, 0<p<1$. The spaces $\operatorname{Re} H^{p}$ are not even function spaces if $0<p<1$. See [6]. It follows from the proofs of Theorems 2.1 and 2.2 that the necessary and sufficient condition given for a distribution to be the distribution of a function in $\operatorname{Re} H^{1}$ is also a necessary and sufficient condition for a distribution 
to be the distribution of $\lim _{t \rightarrow \infty} m_{t}$ for some continuous time, continuous path martingale $m_{t}, 0<t<\infty$, satisfying $E \sup _{t}\left|m_{t}\right|<\infty$.

Now versions of the results just given will be proved for functions defined on Euclidean $n$ space $\mathbf{R}^{n}$. Since the proofs are usually just variations of preceding arguments, we will be brief. Denote Lebesgue measure on $\mathbf{R}^{n}$ by $l^{n}$. Let $\mathbf{R}_{+}^{n+1}=$ $\left\{\left(x_{1}, x_{2}, \ldots, x_{n}, y\right): y>0\right\}$, and write $\left(x_{1}, x_{2}, \ldots, x_{n}, y\right)=(x, y)$. The Poisson kernel for $\mathbf{R}_{+}^{n+1}$ will be written $\boldsymbol{P}_{n}=P$. The formula for this kernel is given by

$$
P(x, y)=\frac{a_{n} y}{\left(y^{2}+|x|^{2}\right)^{(n+1) / 2}}, \quad(x, y) \in \mathbf{R}_{+}^{n+1},
$$

where $a_{n}=\pi^{-(n+1) / 2} \Gamma[(n+1) / 2]$. The function $f\left(x_{1}, x_{2}, \ldots, x_{n}\right)$ will always be an integrable real valued function on $\mathbf{R}^{n}$, and the harmonic function $u_{f}=u$ is defined on $\mathbf{R}_{+}^{n+1}$ by

$$
u(x, y)=\int_{\mathbf{R}^{n}} f(x-t) P(t, y) d l^{n}(t)
$$

The cone in $\mathbf{R}_{+}^{n+1}$ with vertex at $(x, 0)$ and with unit thickness is denoted by $\Gamma(x)$, so that

$$
\Gamma(x)=\{(s, y): 0<y<\infty \text { and }|x-s|<y\} .
$$

The nontangential maximal function of $f$ is defined by

$$
N_{f}(x)=N(x)=\sup _{(s, y) \in \Gamma(x)}|u(s, y)| .
$$

Burkholder, Gundy, and Silverstein have shown in [4] that, if $f$ is an integrable function on the real line,

$$
c_{p}\|f\|_{H^{p}}^{p}<\int_{-\infty}^{\infty} N(x)^{p} d x<C_{p}\|f\|_{H^{p}}^{p}, \quad 0<p<\infty
$$

where $\|f\|_{H^{p}}^{p}=\int_{-\infty}^{\infty}|f(x)+i \tilde{f}(x)|^{p} d x, \tilde{f}$ being the Hilbert transform of $f$. It is generally accepted to say that if $f$ is defined on $\mathbf{R}^{n}, n>1$, then $f \in H^{p}\left(\mathbf{R}^{n}\right)$ if and only if $\int_{\mathbf{R}^{n}} N(x)^{p} d l^{n}(x)$ is finite and this is the definition of $H^{p}\left(\mathbf{R}^{n}\right)$ used here.

Let $f_{\delta}(x),-\infty<x<\infty$, be the function defined on the real line which is nonnegative and not increasing on $(0, \infty)$, not positive and not increasing on $(-\infty, 0)$, and which has the same distribution as $f$, that is

$$
l^{n}\left\{x \in \mathbf{R}^{n}: f(x) \in A\right\}=l^{1}\left\{x \in \mathbf{R}^{1}: f_{\delta}(x) \in A\right\} \text { for all Borel sets } A .
$$

If $n=1$, we call $f_{\delta}$ a rearrangement of $f$, but if $n>1$ we do not, reserving this term for functions defined on the same space, $\mathbf{R}^{n}$, that $f$ is defined on. Define $M(x)=$ $\int_{-x}^{x} f_{\delta}(t) d t$, and

$$
A_{p}(f)=\sum_{n=-\infty}^{\infty} 2^{n(1-p)} M\left(2^{n}\right), \quad 0<p<\infty
$$

The following theorem will be proved. 
THEOREM 2.3. Let $f$ be an integrable real valued function on the real line. Then

$$
\int_{0}^{\infty}|M(x) / x|^{p} d x<C_{p}\|f\|_{H^{p}}^{p}, \quad 0<p<1,
$$

and

$$
\left\|f_{\delta}\right\|_{H^{p}}^{p} \leqslant C_{p}\left(\|f\|_{p}^{p}+\int_{0}^{\infty}|M(x) / x|^{p} d x\right), \quad 1 / 2<p<1 .
$$

The following theorem extends this to higher dimensions.

THEOREM 2.4. Let $f$ be an integrable real valued function on $\mathbf{R}^{n}, n \geq 1$. Then

$$
\int_{0}^{\infty}|M(x) / x|^{p} d x<C_{p, n} \int_{\mathbf{R}^{n}} N_{f}(x)^{p} d l^{n}(x), \quad 0<p<1 .
$$

If $n /(n+1)<p<1$, there is some rearrangement $g$ of $f$ satisfying

$$
\int_{\mathbf{R}^{n}} N_{g}(x)^{p} d l^{n}(x)<C_{p, n}\left(\|f\|_{p}^{p}+\int_{0}^{\infty}|M(x) / x|^{p} d x\right) .
$$

Note that (2.12) in case $n=1$, together with (2.9), implies (2.10).

The proof of the following lemma is similar to the proof of Lemma 2.1 and is omitted.

LEMMA 2.3. If $f$ is an integrable function on $\mathbf{R}^{n}$ then

$$
c_{p} A_{p}(f)-C_{p}\|f\|_{p}^{p} \leqslant \int_{0}^{\infty}|M(x) / x| d x \leqslant K_{p}\left(A_{p}(f)+\|f\|_{p}^{p}\right) .
$$

Now (2.12) will be proved. Let $X(t)=\left(X_{1}(t), \ldots, X_{n}(t), X_{n+1}(t)\right)$ be standard $n+1$ dimensional Brownian motion. Let $T_{y}=\inf \left\{t>0: X_{n+1}(t)=y\right\}$ : and shorten $T_{0}$ to $T$. If $(x, y) \in \mathbf{R}^{n+1}$ let the subscripts on $P_{(x, y)}$ and $E_{(x, y)}$ denote starting position for $X(t)$, as before. Extend $u^{f}$ to $(x, 0) \in \mathbf{R}^{n}$ by defining $u^{f}(x, 0)=f(x)$, and define $u^{*}=\sup _{0<t<T} u(X(t))$. Burkholder and Gundy show in [5] that

$$
\begin{aligned}
c_{p, n} \int_{\mathbf{R}^{n}} N(x)^{p} d l^{n}(x) & <\sup _{y>0} \int_{\mathbf{R}^{n}} E_{(x, y)}\left(u^{*}\right)^{p} d l^{n}(x) \\
& \leqslant C_{p, n} \int_{\mathbf{R}^{n}} N(x)^{p} d l^{n}(x) .
\end{aligned}
$$

Now

$$
\left(s^{n} / a_{n}\right) P(x, s) \nearrow 1 \text { as } s \rightarrow \infty \text { if } x \in \mathbf{R}^{n} .
$$

Since $X(t)$ eventually hits each set of the form $\left\{(x, y): x \in \mathbf{R}^{n}\right\}$, the strong Markov property can be used to show $\int_{\mathbf{R}^{n}} E_{(x, y)}\left(u^{*}\right)^{p} d l^{n}(x)$ is not decreasing as $y$ increases, proving the first inequality in the expression

$$
\begin{aligned}
\sup _{y>0} \int_{\mathbf{R}^{n}} E_{(x, y)}\left(u^{*}\right)^{p} d l^{n}(x) & =\lim _{y \rightarrow \infty} \int_{\mathbf{R}^{n}} E_{(x, y)}\left(u^{*}\right)^{p} d l^{n}(x) \\
& =\lim _{s \rightarrow \infty}\left(s^{n} / a_{n}\right) E_{(0, s)}\left(u^{*}\right)^{p} .
\end{aligned}
$$

To prove the second equality, note that, since $f$ is integrable, $\sup _{x \in R^{n}}|u(x, y)| \rightarrow 0$ as $y \rightarrow \infty$. Thus $E_{(0, s)}\left(u^{*}\right)^{p}$ is just about equal to $E_{(0, s)} \sup _{T_{\sqrt{s}}<t<T}|u(X(t))|^{p}$ if $s$ is large, and since $X(T \sqrt{s})$ has density $P(x, s-\sqrt{s})$ at the point $(x, \sqrt{s})$ under $\boldsymbol{P}_{(0, s)},(2.14)$ and the strong Markov property give the result. 
Let $\alpha_{\lambda}=\left\{\sup _{r>0}: f_{\delta}(r)>\lambda\right\}$, and let $\beta_{\lambda}$ be the unique negative number such that $\int_{\beta_{\lambda}}^{\alpha_{\lambda}} f(x) d x=\lambda\left(\alpha_{\lambda}-\beta_{\lambda}\right)$.

Then, using (2.14), the argument of the first section can be adapted to these circumstances to yield

$$
\lim _{s \rightarrow \infty}\left(s^{n} / a_{n}\right) P_{(0, s)}\left(u_{+}^{*}>\lambda\right)>\alpha_{\lambda}-\beta_{\lambda},
$$

so that

$$
\begin{aligned}
\lim _{s \rightarrow \infty}\left(s^{n} / a_{n}\right) E_{(0, s)}\left(u_{+}^{*}\right)^{p} & >p \int_{0}^{\infty}\left(\alpha_{\lambda}-\beta_{\lambda}\right) \lambda^{p-1} d \lambda \\
& >c_{p} \sum_{n=-\infty}^{\infty}\left(\int_{-2^{n}}^{2^{n}} f_{\delta}(x) d x\right)_{+}^{p},
\end{aligned}
$$

for $0<p<\infty$. The analogous inequality for $u_{-}^{*}$, together with (2.13), (2.15), and Lemma 2.3, gives (2.10).

Now (2.11) will be proved. Let

$$
g_{A}=(2 A)^{-1} \int_{-A}^{A} f_{\delta}(x) d x
$$

and let

$$
h_{A}(x)=\left(f_{\delta}(x)-g_{A}\right) I(-A<x<A) .
$$

Let $m$ be the smallest integer such that $2^{m}>A$. For $n>0$, let $\mathscr{B}_{n}$ be the $\sigma$-algebra of sets in $\left[-2^{m}, 2^{m}\right)$ generated by the interval $\left[-2^{m-n}, 2^{m-n}\right)$, together with the intervals $\left[k 2^{m-n},(k+1) 2^{m-n}\right),-2^{n}<k<2^{n}-1, k \neq-1$ or 0 . Then, with respect to the probability measure $d x / 2^{m+1}$ on $\left[-2^{m}, 2^{m}\right), E\left(h_{A} \mid \mathscr{B}_{n}\right), n>1$, is a martingale. A proof very similar to the proof of Theorem 2.2 yields

$$
\left\|h_{A}\right\|_{H^{p}}^{p}<C_{p}\left(\left\|h_{A}\right\|_{p}^{p}+A_{p}\left(h_{A}\right)\right), \quad 1 / 2<p<1 .
$$

We remark that

$$
\int_{-2^{n}}^{2^{n}} h_{A}(x) d x=0 \text { if } n>m .
$$

Next, (2.11) will be derived from (2.16) via a limiting argument. Since $\lim _{A \rightarrow \infty} \tilde{h}_{A}(x)=\tilde{f}_{\delta}(x)$,

$$
\lim _{A \rightarrow \infty}\left\|h_{A}\right\|_{H^{p}}^{p}>\left\|f_{\delta}\right\|_{H^{p}}^{p}, \quad 0<p<\infty .
$$

Since clearly $A_{p}(f)=\infty$ if $1 / 2<p<1$ and $\int_{-\infty}^{\infty} f(x) d x \neq 0$, to prove (2.11) it is sufficient to show

$$
\begin{aligned}
\lim _{A \rightarrow \infty}\left\|h_{A}\right\|_{p} & =\|f\|_{p}, \quad \text { if }\|f\|_{p}<\infty \quad \text { and } \\
\int_{-\infty}^{\infty} f(x) d x & =0, \quad 1 / 2<p<1,
\end{aligned}
$$

and

$$
\begin{aligned}
& \lim _{A \rightarrow \infty} A_{p}\left(h_{A}\right)=A_{p}(f), \quad \text { if }\|f\|_{p}<\infty \text { and } \\
& \int_{-\infty}^{\infty} f(x) d x=0, \quad 1 / 2<p<1 .
\end{aligned}
$$


Now $\|f\|_{p}<\infty$ implies $\left|f_{\delta}(x)\right|=o\left(|x|^{-1 / p}\right)$ as $|x| \rightarrow \infty$. If in addition we have $\int_{-\infty}^{\infty} f(x) d x=\int_{-\infty}^{\infty} f_{\delta}(x) d x=0$, then

$$
\begin{aligned}
\left|\int_{-A}^{A} f_{\delta}(x) d x\right| & \leqslant \int_{-\infty}^{-A}\left|f_{\delta}(x)\right| d x+\int_{A}^{\infty}\left|f_{\delta}(x)\right| d x \\
& =o\left(A^{(p-1) / p}\right) \text { as } A \rightarrow \infty, 0<p<1,
\end{aligned}
$$

and, using this estimate, (2.18) and (2.19) can be proved for the exponents $1 / 2<p<1$. For $p=1$, the estimate $\int_{-A}^{A} f_{\delta}(x) d x \rightarrow 0$ suffices.

Finally, we verify the last sentence in the statement of Theorem 2.4. For $n=1$ it is implied by (2.11) and (2.9). Let $n$ be a fixed integer exceeding 1 , and if $\alpha>0$ let $Q_{\alpha}$ be the cube in $\mathbf{R}^{n}$ with center 0 , edges parallel and perpendicular to the coordinate axes, and edge length $\alpha$, and let $S_{\alpha}$ be the surface of $Q_{\alpha}$. If $f$ is an integrable function on $\mathbf{R}^{n}$, let $f_{a}$ be the rearrangement of $f$ which is constant and not negative on each set of the form $\mathbf{R}_{+}^{n} \cap S_{\alpha}$, constant and not positive on $\left(\mathbf{R}^{n}-\mathbf{R}_{+}^{n}\right) \cap S_{\alpha}$, and such that the values of $f_{a}$ on $\mathbf{R}_{+}^{n} \cap S_{\alpha}$ are not increasing as $\alpha$ increases from 0 to $\infty$, and the values of $f_{a}$ on $\left(\mathbf{R}_{n}-\mathbf{R}_{n}^{+}\right) \cap S_{\alpha}$ are not decreasing as $\alpha$ increases from 0 to $\infty$. Let, for each $A>0, \varphi_{A}=A^{-n} \int_{Q_{A}} f_{a}(x) d l^{n}(x)$, and let $\gamma_{A}(x)=\left(f_{a}(x)-\varphi_{A}\right) I\left(x \in Q_{A}\right)$. Let $m$ be the smallest integer such that $2^{m}>A$. Let $\mathscr{B}_{0} \subset \mathscr{B}_{1} \subset \mathscr{B}_{2} \subset$ be a sequence of $\sigma$-fields of sets contained in $Q_{2^{m}}$ with the properties

(i) $\mathscr{B}_{i}$ is generated by a finite disjoint collection $\mathcal{C}_{i}$ of "rectangles", that is, sets of the form $I_{1} \times I_{2} \times \cdots \times I_{n}$, where each of the $I_{j}$ are intervals,

(ii) $Q_{2^{(m n-k) / n}} \in \mathcal{C}_{k}$,

(iii) the maximum diameter of all the rectangles in $C_{i}$ goes to 0 as $i$ goes to infinity,

(iv) each of the rectangles in $\mathcal{C}_{i+1}$ is contained in a rectangle in $\mathcal{C}_{i}$ of no more than $\eta$ times its measure, where $\eta$ is a positive number depending on $n$ but not on $i$ or $m$, and

(v) the ratio of the length of the maximum side to the length of the minimum side of the rectangles in $\mathcal{C}_{i}$ is bounded by a constant depending on $n$ but not on $i$ or $m$.

Then, with this setup, a proof that

$$
\int_{\mathbf{R}^{n}} N_{f_{a}}(x)^{p} d l^{n}(x)<C_{p, n}\left(\|f\|_{p}^{p}+A_{p}(f)\right)
$$

for the exponents $n /(n+1)<p<1$ can be made in a manner very similar to the proof of $(2.11)$ for $1 / 2<p<1$. The reason that $p$ may only go down to $n /(n+1)$ is that atoms, if we define them like we did before (2.7), may not be in $H^{p}$ for $p<n /(n+1)$, but must be if $p>n /(n+1)$. See [6]. Note that

$$
A_{p}(f)=\sum_{k=-\infty}^{\infty} 2^{k(1-p)}\left|\int_{Q 2^{k+1) / n}} f_{a}(x) d l^{n}(x)\right|^{p} .
$$

3. Rotations of functions. Let $\mathbb{Q}_{0} \subset \mathbb{Q}_{1} \subset \mathbb{Q}_{2} \subset$ be a sequence of $\sigma$-fields of the Borel sets of $T$ such that $\mathbb{Q}_{0}=\{\phi, T\}$, each $\mathbb{Q}_{i}$ is generated by a collection $\mathcal{C}_{i}$ of disjoint arcs, and each set in $\mathbb{Q}_{i+1}$ is contained in a set in $\mathbb{Q}_{i}$ of no more than $\delta$ its 
measure, where $\delta$ is a fixed positive number not depending on $i$. Such a sequence of $\sigma$-fields will be called $\delta$-regular arc generated. The dyadic $\sigma$-fields are 2-regular arc generated, and the $\sigma$-fields $\mathscr{F}_{n}$ of the last section are 4-regular arc generated. An integrable function $f$ on $T$ is said to be in $H^{P}\left(\mathbb{Q}_{n}, n>0\right)$ if and only if $E \sup _{n}\left|E\left(f \mid \mathbb{Q}_{n}\right)\right|^{p}<\infty$. It is known that if $\mathbb{Q}_{n}, n>0$, is $\delta$-regular arc generated and that if $\sigma\left(\mathbb{Q}_{n}, n \geqslant 0\right)$ contains all the Borel subsets of $T$, then if a function is in $H^{1}\left(Q_{n}, n>0\right)$ it is in $\operatorname{Re} H^{1}$, as was shown for a special case in the last section. The converse is not true, but a weaker related result is. Let $f_{r}\left(e^{i \theta}\right)=f\left(e^{i(\theta-r)}\right)$ be the rotation of $f$ through the angle $r$. The following theorem is proved

THEOREM 3.1. Let $\mathbb{Q}_{n}, n \geqslant 1$, be a $\delta$-regular arc generated sequence of $\sigma$-fields of Borel sets of $T$. Let $f$ be an integrable function on $T$. Then

$$
\int_{0}^{2 \pi}\left(\int_{T} \sup _{n}\left|E\left(f_{\theta} \mid \mathbb{Q}_{n}\right)\right|^{p} d m\right) d \theta<C_{p, \delta}\|f\|_{H^{p}}^{p}, \quad 1 / 2<p<1 .
$$

Thus if $f \in H^{1}$ almost every rotation of $f$ is in $H^{1}\left(Q_{n}, n>0\right)$.

Proof of Theorem 3.1. If $f \in \operatorname{Re} H^{1}$ a theorem of C. Fefferman (for $p=1$ ) and of Coifman (see [6]) permits us to write $f=\Sigma \lambda_{i} a_{i}$, where the $\lambda_{i}$ are real numbers, the $a_{i}$ are functions supported on an arc of length $\varepsilon_{i}$ satisfying $\left|a_{i}\right|<\varepsilon_{i}^{-1}$ and $\int_{T} a_{i}=0$, and

$$
\sum\left(\lambda_{i} \varepsilon_{i}^{-1}\right)^{p} \varepsilon_{i} \leqslant C_{p}\|f\|_{H^{p}}^{p}, \quad 1 / 2<p<1 .
$$

This decomposition is perhaps different if the $p$ are different. Thus, to prove (3.1) when $1 / 2<p \leqslant 1$, it suffices to prove it only for functions having the form of the $a_{i}$. This will now be done.

Let $\varepsilon>0$, and let $\Gamma$ be an arc such that $m(\Gamma)=\varepsilon$. Let $g$ be a function supported on $\Gamma$ which satisfies $\int_{T} g d m=0$ and $|g|<\varepsilon^{-1}$. To complete the proof of Theorem 3.1 it will be shown that

$$
\int_{0}^{2 \pi}\left(\int_{T} \sup _{n}\left|E\left(g_{\theta} \mid \mathbb{Q}_{n}\right)\right|^{p} d m\right) d \theta<C_{p, \delta} \varepsilon^{1-p}, \quad 1 / 2<p<1 .
$$

For the rest of this section $p$ will be a number in $(1 / 2,1]$. If $J$ is one of the arcs generating $\mathcal{Q}_{n}$,

$$
\left|E\left(g_{\theta} \mid Q_{n}\right)\right| I(J)=\left|m(J)^{-1} \int_{J} g_{\theta} d m\right| I(J)=h(J, \theta) .
$$

Let $\mathscr{R}=\cup_{i=0}^{\infty} \mathcal{C}_{i}$ be the collection of all the arcs generating all the $\mathbb{Q}_{i}$. Then

$$
\sup _{n}\left|E\left(g_{\theta} \mid \mathcal{Q}_{n}\right)\right|=\sup _{J \in \mathscr{T}} h(J, \theta) .
$$

Divide $\mathscr{K}=\mathcal{S} \cup \mathcal{L}$, where $\mathcal{L}$ is all those $\operatorname{arcs} J$ in $\mathscr{N}$ such that $m(J)>\varepsilon$ and $\mathcal{S}$ is all those such that $m(J)<\varepsilon$. Let $\Gamma_{\theta}$ be the arc $\Gamma$ rotated through the angle $\theta$. Then $g_{\theta}$ is supported by $\Gamma_{\theta}$. Let $\hat{\Gamma}_{\theta}$ be the arc with the same center as $\Gamma_{\theta}$ and length

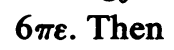

$$
\sup _{J \in \delta} h(J, \theta)<\varepsilon^{-1} I\left(\hat{\Gamma}_{\theta}\right)
$$


since conditional expectation decreases the $L^{\infty}$ norm, and any arc in $\mathcal{S}$ which intersects $\Gamma_{\theta}$ is contained in $\hat{\Gamma}_{\theta}$. Thus

$$
\int_{0}^{2 \pi}\left(\int_{T} \sup _{J \in \delta}|h(J, \theta)|^{p} d m\right) d \theta<\int_{0}^{2 \pi}\left(\varepsilon^{-p} \cdot 3 \varepsilon\right) d \theta=6 \pi \varepsilon^{1-p} .
$$

Now, for any $\operatorname{arc} J, \int_{J} g_{\theta} d m=0$ unless $\theta$ is within $\pi \varepsilon$ of one of the endpoints of $J$, a set of $\theta$ of length $4 \pi \varepsilon$, and in any event $\left|\int_{J} g_{\theta} d m\right|<1$, implying $\int_{T}|h(J, \theta)|^{p} d m \leqslant m(J)^{1-p}$ so that

$$
\int_{0}^{2 \pi}\left(\int_{T}|h(J, \theta)|^{p} d m\right) d \theta<4 \pi \varepsilon m(J)^{1-p} .
$$

Now each point of $T$ is contained in at most one arc $J$ of $\Re$ satisfying $x(1-\delta)<m(J)<x$, for any positive number $x$, so that there are at most $[x(1-\delta)]^{-1}$ arcs in $\Re$ with measure falling in this range. Let $N$ be the integer satisfying $(1-\delta)^{N+1}<\varepsilon<(1-\delta)^{N}$, and let $\mathfrak{N}_{i}$ be those arcs $J$ in $\Re$ such that $(1-\delta)^{i+1}<m(J)<(1-\delta)^{i}$. Then $\mathcal{L} \subseteq \cup \cup_{i=0}^{N} \Re_{i}$, and if $\left|T_{i}\right|$ is the number of arcs in $\mathfrak{N}_{i}$,

$$
\begin{aligned}
\int_{0}^{2 \pi}\left(\int_{T} \sup _{J \in \mathcal{L}}|h(J, \theta)|^{p}\right. & d m) d \theta \leqslant \sum_{J \in \mathcal{E}} \int_{0}^{2 \pi}\left(\int_{T}|h(J, \theta)|^{p} d m\right) d \theta \\
& \leqslant \sum_{i=0}^{N}\left(\sum_{J \in \Im \mathbb{R}_{i}} \int_{0}^{2 \pi}\left(\int_{T}|h(J, \theta)|^{p} d m\right) d \theta\right) \\
& \leqslant \sum_{i=0}^{N}\left|\Re_{i}\right| 4 \pi \varepsilon(1-\delta)^{i(1-p)} \\
& \leqslant \sum_{i=0}^{N}(1-\delta)^{-(i+1)} 4 \pi \varepsilon(1-\delta)^{i(1-p)}=C_{p, \delta} \varepsilon \sum_{i=0}^{N}(1-\delta)^{-i p} \\
& =C_{p, \delta} \varepsilon(1-\delta)^{-N p}<C_{p, \delta} \varepsilon^{1-p}
\end{aligned}
$$

using inequality (3.5) to derive the third inequality above. This, together with (3.3) and (3.4), establishes (3.2).

4. The extremal rearrangements. This section is primarily devoted to the proof of Theorem 1.2. First a related question concerning stopping times for Brownian motion is answered. The way Green functions are used was suggested by Baernstein's nonprobabilistic treatment of the symmetric decreasing rearrangement, in [2]. Notation remains as before. The area of a set $A \subset \mathbf{C}$ is written $\sigma(A)$, and if $R$ is a connected region in $C$ let $G(R, z, w)$ stand for the Green function of $R$ and define $\tau_{R}=\inf \left\{t \geqslant 0: Z_{t} \notin R\right\}$. G. Hunt shows in [11] that if $A$ is a measurable subset of $R$ and $w \in R$ then

$$
E_{w} \int_{0}^{\tau_{R}} I\left(Z_{t} \in A\right) d t=\int_{A} G(R, z, w) d \sigma(z) .
$$

A nonnegative random variable $T$ will be called a Markov time for $Z_{t}$ if $Z_{T+t}-Z_{T}, t>0$, is a standard Brownian motion independent of $Z_{t}, 0<t<T$. This is a generalization of stopping times to a class of random variables for which 
the strong Markov property still holds. Let $a<0<b$ be fixed real numbers and let $S$ be the strip $\{x+i y: a<x<b\}$. Let $\tau<\tau_{S}$ be a Markov time for $Z_{t}$. Then, by (4.1) and the strong Markov property, if $A$ is a measurable set contained in $S$,

$$
\begin{aligned}
E \int_{0}^{\tau} I\left(Z_{t} \in A\right) d t & =E \int_{0}^{\tau_{S}} I\left(Z_{t} \in A\right) d t-E \int_{\tau}^{\tau_{S}} I\left(Z_{t} \in A\right) d t \\
& =\int_{A} G(S, z, 0) d \sigma(z)-E E_{Z_{\tau}} \int_{0}^{\tau_{S}} I\left(Z_{t} \in A\right) d t \\
& =\int_{A} G(S, z, 0) d \sigma(z)-\int_{S}\left[\int_{A} G\left(S, z, Z_{\tau}\right) d \sigma(z)\right] d P\left(Z_{\tau}\right) \\
& =\int_{A}\left[G(S, z, 0)-\int_{S} G\left(S, z, Z_{\tau}\right) d P\left(Z_{\tau}\right)\right] d \sigma(z)
\end{aligned}
$$

The quantity in brackets in the last expression will be denoted $G_{\tau}(z)$, so that

$$
E \int_{0}^{\tau} I\left(Z_{t} \in A\right) d t=\int_{A} G_{\tau}(z) d \sigma(z)
$$

Considered as a function of $z, G(S, z, w)$ is harmonic in $S$ except for a pole at $w$, so $G$ is subharmonic in $S-\{0\}$ and is harmonic in any region $R \subset S$ satisfying $P\left(Z_{\tau} \in R\right)=0$. We extend $G_{\tau}$ continuously to $C-S$ by defining it to be zero there, and remark that if a region $R$ is formed by removing line segments from $S$, then (4.1), and elementary properties of Green functions, imply that $G_{\tau_{R}}(z)=0$ on $S-R$.

Now let $s>0$, and define

$$
\begin{aligned}
h_{s}(z) & =h_{s}(x+i y)=h_{s}(x, y)=(x-s)^{2} / 2 s \quad \text { if }|x|<s, \\
h_{s}(x, y) & =|x|-(s / 2) \quad \text { if }|x| \geqslant s .
\end{aligned}
$$

Let $f$ be any of the functions $|z|^{p}, p>0,|y|^{p}, p>1$, or $h_{s}(x-\gamma+i y),-\infty<\gamma$ $<\infty, s>0$. Let $\nabla^{2}$ be the Laplacian and define

$$
M_{t}=f\left(Z_{t}\right)-\frac{1}{2} \int_{0}^{t} \nabla^{2} f\left(Z_{s}\right) d s, \quad 0<t<\infty .
$$

Then Ito's lemma implies that $M_{t}, 0<t<\infty$, is a martingale. It is not difficult to show that, under $P$, $\sup _{t>0} E_{\min \left(t, \tau_{s}\right)}^{2}<\infty$, so that if $\tau$ is a Markov time not exceeding $\tau_{S}, E M_{\tau}=M_{0}=f(0)$, implying

$$
E f\left(Z_{\tau}\right)=\frac{1}{2} \int_{0}^{\tau} \nabla^{2} f\left(Z_{s}\right) d s+f(0) .
$$

This and the extension of (4.2) from indicator functions to $\nabla^{2} f$ gives

$$
E f\left(Z_{\tau}\right)=\frac{1}{2} \int_{S} \nabla^{2} f(z) G_{\tau}(z) d \sigma(z)+f(0)
$$

For $f=h_{s}(x-\gamma+i y)$, (4.3) becomes

$$
E h_{s}\left(X_{\tau}-\gamma+i Y_{\tau}\right)=\frac{1}{2 s} \int_{-\infty}^{\infty} d y \int_{\gamma-s}^{\gamma+s} G_{\tau}(x+i y) d x+h_{s}(-\gamma) \text {, }
$$


and letting $s$ decrease to 0 in this expression yields

$$
E\left|X_{\tau}-\gamma\right|=\int_{-\infty}^{\infty} G_{\tau}(\gamma+i y) d s+|\gamma|
$$

The Green functions $G(S, z, w)$ are readily calculated. Considered as a function of $z$, with $w$ fixed, they have a logarithmic pole at $w$, and $G(S, x+i y, w)=$ $o\left(e^{-\alpha|y|}\right)$ as $|y| \rightarrow \infty$, for a constant $\alpha$ depending on $a$ and $b$. Let $S^{+}=S \cap\{y>$ $0\}$, and define, for each point $z=x+i y \in \overline{S^{+}}$,

$$
A_{\tau}(z)=\int_{y}^{\infty} G_{\tau}(x+i \eta) d \eta+\int_{-\infty}^{-y} G_{\tau}(x+i \eta) d \eta .
$$

Now $G_{\tau}(z)<G(S, z, 0)$, implying $A_{\tau}(z)$ is bounded in $\bar{S}^{+}$, and it is not difficult to show that if $h_{w}(z), w, z \in S$, is defined by

$$
h_{w}(z)=h_{w}(x+i y)=\int_{y}^{\infty} G(S, x+i s, w) d s
$$

then the family of functions $h_{w}(z), w \in S$, is equicontinuous in $S$, and this, together with the definition of $G_{\tau}$, shows that $A_{\tau}$ is continuous in $\bar{S}^{+}$.

Since $G_{\tau}$ is subharmonic in $S, G_{\tau}$ is no larger at a point of $S$ than its average over a disc contained in $S$ which is centered at that point. Thus if $B(z)$ is defined for $z=x+i y \in S$ by

$$
B(z)=\int_{y}^{\infty} G_{\tau}(x+i \eta) d \eta,
$$

and if $x+i y$ is a point in $S$ at least a distance $r>0$ from the boundary,

$$
\begin{aligned}
B(z) & =\int_{0}^{\infty} G_{\tau}(x+i(y+\eta)) d \eta \\
& >\int_{0}^{\infty}\left[\left(\pi r^{2}\right)^{-1} \int_{-r}^{r} d s \int_{-\sqrt{r^{2}-s^{2}}}^{\sqrt{r^{2}-s^{2}}} G_{\tau}((x-s)+i(y+\eta-t)) d t\right] d \eta \\
& =\left(\pi r^{2}\right)^{-1} \int_{-r}^{r} d s \int_{-\sqrt{r^{2}-s^{2}}}^{\sqrt{r^{2}-s^{2}}}\left(\int_{0}^{\infty} G_{\tau}((x-s)+i(y-t+\eta)) d \eta\right) d t \\
& =\left(\pi r^{2}\right)^{-1} \int_{-r}^{r} d s \int_{-\sqrt{r^{2}-s^{2}}}^{\sqrt{r^{2}-s^{2}}} B(x-s+i(y-t)) d t .
\end{aligned}
$$

Thus, $B$ itself is subaveraging and so is subharmonic. If $G$ is harmonic on $\cup_{\eta>y}\{z$ : $|z-(x+i \eta)|<r\}$ then the inequality in the above expression is an equality, and $B$ is harmonic at $x+i y$. Thus $A_{\tau}$ is subharmonic in $S^{+}$, and perhaps harmonic at some points of $S^{+}$.

Now let $a=s_{1}<s_{2}<\cdots<s_{n}=b$ be $n$ nonzero real numbers and let $p_{i}$, $1<i<n$, be $n$ positive numbers such that $\sum_{i=1}^{n} p_{i}=\sum p_{i}=1$ and $\sum p_{i} s_{i}=0$. Let $R(s, p)$ be the region

$$
S-\bigcup_{i=2}^{n-1}\left\{x+i y: x=s_{i},|y|<\gamma_{i}\right\},
$$

where $\gamma_{i}, 2<i<n-1$, are the unique positive numbers such that

$$
P\left(Y_{\tau_{R(s, p)}}=s_{i}\right)=p_{i}, \quad 1<i<n .
$$


To prove that there is a unique set of numbers $\gamma_{i}$ satisfying these requirements, we first note that it is not hard to show that there is a set of numbers $c_{i}, 2<i<n-1$, such that if $\tau=\inf \left\{t: Y_{t} \notin S-\left\{x+i y: x=s_{i},|y|<c_{i}\right\}\right\}$, then $\sum_{i=2}^{n-1} \mid P\left(X_{\tau}=s_{i}\right)$ $-p_{i} \mid$ is as small as possible for stopping times of this form, and then it can be argued that, if this sum is not zero, one of the $c_{i}$ could be increased or decreased a very small amount to make the sum smaller. Thus $P\left(X_{\tau}=s_{i}\right)=p_{i}, 2<i<n-1$, and, since $E X_{\tau}=0$, we must also have $P\left(X_{\tau}=s_{i}\right)=p_{i}, i=1, n$. We take the numbers $c_{i}$ for our $\gamma_{i}$. To show they are unique, note that if

$$
T=\min \left(\tau_{s}, \inf \left\{t: X_{t}=s_{i},\left|y_{i}\right|<\delta_{i} \text { for some } i, 2<i<n-1\right\}\right),
$$

and if $A=\left\{i: \delta_{i}>\gamma_{i}\right\}$ and $B=\left\{i: \delta_{i}<\gamma_{i}\right\}$, then clearly

$$
\begin{aligned}
& \sum_{i \in A} P\left(X_{T}=s_{i}\right)>\sum_{i \in A} P\left(X_{\tau}=s_{i}\right) \text { and } \\
& \sum_{i \in B} P\left(X_{T}=s_{i}\right)<\sum_{i \in B} P\left(X_{\tau}=s_{i}\right) .
\end{aligned}
$$

Now let the numbers $s_{i}$ be as above and let $q_{i}, 1<i<n$, be $n$ positive numbers such that $0<\Sigma q_{i}<1$ and $\Sigma q_{i} s_{i}=0$. Let $T(s, q)$ be the randomized Markov time constructed in the following way. Flip a coin with probability $\Sigma q_{i}$ of heads. If the coin is tails, $T(s, q)=0$, and if the coin is heads, $T(s, q)=\tau_{R(s, p)}$, where $p_{i}=$ $q_{i} / \Sigma q_{i}$. Let $\mathcal{T}(s, q)=\mathcal{T}$ be the collection of all Markov times $\tau<\tau_{s}$ satisfying

$$
P\left(X_{\tau}=0\right)=1-\sum q_{i} \text { and } P\left(X_{\tau}=s_{i}\right)=q_{i}, \quad 1<i<n .
$$

Then $T(s, q) \in \mathcal{T}$, and is in some ways extremal, as the following theorem shows.

TheOrem 4.1. Let $\tau \in \mathcal{T}(s, q)$. Then

$$
\begin{array}{ll}
E\left|Y_{T(s, q)}\right|^{\rho}<E\left|Y_{\tau}\right|^{\rho}, & 1<\rho<2, \\
E\left|Y_{T(s, q)}\right|^{\rho}>E\left|Y_{\tau}\right|^{\rho}, & 2<\rho<\infty, \\
E\left|Z_{T(s, q)}\right|^{\rho}<E\left|Z_{\tau}\right|^{\rho}, & 0<\rho<2, \\
E\left|Z_{T(s, q)}\right|^{\rho}>E\left|Z_{\tau}\right|^{\rho}, & 2<\rho<\infty .
\end{array}
$$

Proof. We shorten $T(s, q)$ to $T$. The function $A_{T}$ is continuous in $\overline{R(s, p)^{+}}$, harmonic in $R(s, p)^{+}$, and vanishes at infinity. Also,

$$
A_{T}(a+i y)=A_{T}(b+i y)=0
$$

for each $y \geqslant 0$, and, by (4.4),

$$
A_{T}(\gamma)=E\left|X_{T}-\gamma\right|-|\gamma|=\sum p_{i}\left|s_{i}-\gamma\right|-|\gamma|, \quad-\infty<\gamma<\infty .
$$

Since $G_{T}$ vanishes on $\left\{s_{i}+i y: 0<|y|<\gamma_{i}\right\}$, this equality implies

$$
A_{T}\left(s_{i}+i y\right)=\sum_{j=1}^{n} p_{j}\left|s_{j}-s_{i}\right|-\left|s_{i}\right|, \quad 0<y<\gamma_{i},
$$

completing the description of the boundary values of $A_{T}$. Now $A_{\tau}$ is subharmonic in $R(s, p)^{+}$, and has the same boundary values, with the possible exception of the boundary points $s_{j}+i y, 0<y<\gamma_{j}, 2<j<n-1$. Since $G_{\tau}$ does not necessarily 
vanish on $\left\{s_{j}+i t, 0 \leqslant t \leqslant \gamma_{j}\right\}, A_{\tau}\left(s_{j}+i y\right)$ may be smaller than $A_{T}\left(s_{j}+i y\right)$. Thus $A_{\tau}-A_{T}$ is bounded and subharmonic in $R(s, p)^{+}$, and has nonpositive boundary values, implying it is nonpositive everywhere, so that

$$
A_{\tau}(z) \leqslant A_{T}(z), \quad z \in \overline{R(s, p)^{+}} .
$$

By (4.3), for $g(z)=|y|^{p}, p>1$, or $g(z)=|z|^{p}, p>0$, we have

$$
\begin{aligned}
E g\left(Z_{\tau}\right) & =\frac{1}{2} \int_{a}^{b} d x \int_{-\infty}^{\infty} \nabla^{2} g(x+i y) G_{\tau}(x+i y) d y \\
& =-\frac{1}{2} \int_{a}^{b} d x \int_{0}^{\infty} \nabla^{2} g(x+i y) \frac{\partial}{\partial y} A_{\tau}(x+i y) d y,
\end{aligned}
$$

with the corresponding formula when $T$ replaces $\tau$. Note that $\nabla^{2}|y|^{p}$ is decreasing as $y$ increases for $1<p<2$ and increasing if $p>2$, and $\nabla^{2}|x+i y|^{p}$ is, if $x$ is fixed, decreasing as $|y|$ increases for $0<p<2$, and increasing if $p>2$. This, (4.9), (4.10), and the facts that $A_{\tau}(x)=A_{T}(x), a<x<b$, and that both $A_{\tau}$ and $A_{T}$ go to zero as $y \rightarrow \infty$, can be used together with integration by parts to prove all of Theorem 4.1 except (4.5) for the exponent $p=1$. This case follows from the truth of (4.5) when $\rho>1$, via a limiting argument.

It can be shown that the times $T(s, q)$ are the unique times in $\mathcal{T}(s, q)$ which are extremal in the sense of Theorem 4.1, although of course the randomization procedure is arbitrary. For our purposes the following weaker substitute is sufficient.

TheOrem 4.2. Let $0<\Sigma q_{i}<1$. Let $\nu$ be a time in $\mathcal{T}(s, q)$ such that $P(\nu>0)=$ 1. Then strict inequality holds in (4.5), (4.6), (4.7), and (4.8) for $\tau=\nu$.

Proof. The first part of the proof involves carefully going through the proof of Theorem 4.1, showing strict inequality holds at each step for $\nu=\tau$. Since $P\left(Z_{t}=0\right.$ for some $t>0)=0$,

$$
P\left(X_{\nu}=0,\left|Y_{\nu}\right|>0\right)=1-\sum q_{i} .
$$

From this, the definition of $G_{\tau}(z)$, and the fact that $G(S, z, w)$ is superharmonic but not harmonic at $w$, it can be shown that $G_{v}(z)$ is strictly subharmonic in the region $\{x+i y:-\varepsilon<x<\varepsilon\}-\{0\}$, and it follows that $A_{\nu}(z)$ is strictly subharmonic in $R(s, p)^{+}$, for it can be shown that if $P\left(X_{\nu}=0,\left|Y_{p}\right|>\varepsilon\right)>0$ then $A_{\tau}(i \varepsilon)$ is strictly smaller than its average in any disc around $i \varepsilon$. Thus $g(z)=A_{\nu}(z)-A_{T(s, q)}(z)$ is strictly subharmonic and bounded in $R(s, p)^{+}$, and it has already been shown that the boundary values of this function are not positive. Therefore the $g(z)<0$ at some point $z \in R(s, p)^{+}$, and $g(z)<0$ at all points of $R(s, p)^{+}$. This implies $g(z)<0$ at all points of $R(s, p)^{+}$, since $g$ is subharmonic and $R(s, p)^{+}$is connected, for if $g(z)=0$ at some $z \in R(s, p)^{+}$then, by the subaveraging property, $g$ would have to be 0 at almost all points in any disc around $z$ contained in $R(s, p)^{+}$, from which it follows that $g$ would have to be 0 at almost all points of $R(s, p)^{+}$, so that, using the semicontinuity of $g, g$ would be identically zero. Thus

$$
A_{v}(z)<A_{T(s, q)}(z), \quad z \in R(s, p)^{+},
$$


and now the integration by parts argument that concluded the proof of Theorem 4.1 gives strict inequality in (4.5)-(4.8) with $\nu$ in place of $\tau$, with the exception of (4.5) in the case $\rho=1$ which, the reader will recall, was derived by a limiting argument. This case is treated next.

To complete the proof of Theorem 4.2 we show

$$
E\left|Y_{T(s, q)}\right|<E\left|Y_{\nu}\right| \text {. }
$$

First we note that if a random variable $V$ satisfies $P(V>\lambda)=P(V<-\lambda)$ for each $\lambda>0$ and is unbounded and integrable then

$$
E|V+x|-|x|<E|V| \text { if } x>0 \text {. }
$$

Define the time $\eta$ by $\eta=\nu$ on $\left\{X_{\nu} \neq 0\right\}$ and

$$
\eta=\inf \left\{t>\nu: Z_{t}-Z_{\nu} \notin R(s, p)\right\} \quad \text { on }\left\{X_{\nu}=0\right\} \text {. }
$$

Let $p_{i}=q_{i} / \Sigma q_{i}$. Then both $\eta$ and $\tau_{R(s, p)}$ are in $T(s, p)$, and $T(s, p)=\tau_{R(s, p)}$ since $\Sigma p_{i}=1$. Thus, by Theorem 4.1, we have

$$
E\left|Y_{\tau_{R(s, p)}}\right| \leqslant E\left|Y_{\eta}\right| \text {. }
$$

Under $P, Y_{\tau_{R(s,)}}$ is unbounded and symmetrically distributed, so that (4.12) gives the strict inequality in the expression

$$
\begin{aligned}
E\left|Y_{\eta}\right|-E\left|Y_{\nu}\right| & =E\left(\left|Y_{\eta}\right|-\left|Y_{\nu}\right|\right) I(\eta>\nu) \\
& =E\left(E_{Z_{\nu}}\left|Y_{\eta}\right|-\left|Y_{\nu}\right|\right) I(\eta>\nu)=E\left(E\left|Y_{\tau_{R(s, p)}}+Y_{\nu}\right|-\left|Y_{\nu}\right|\right) I(\eta>\nu) \\
& <E\left(E\left|Y_{\tau_{R(s, p)}}\right|\right) I(\eta>\nu)=E \mid Y_{\tau_{R(s, p)}}\left(1-\sum q_{i}\right) .
\end{aligned}
$$

Since $E\left|Y_{T(s, q)}\right|=\left(\Sigma q_{i}\right) E\left|Y_{\tau_{R(s, p)}}\right|$, we have

$$
E\left|Y_{\tau_{R(s, p)}}\right|-E\left|Y_{T(s, q)}\right|>E\left|Y_{\eta}\right|-E\left|Y_{\nu}\right|
$$

and this, together with (4.13), establishes (4.11). A similar approach could have been used to show strict inequality holds in (4.5) and (4.6) for $\nu=\tau$, but not, or at least not as easily, in (4.7) and (4.8).

Let $f, \tilde{f}$, and $F$ be as in $\S 2$. A theorem of P. Lévy says that if $Z_{t}$ starts at 0 then $F\left(Z_{t}\right), 0<t \leqslant \tau_{D}$, is itself Brownian motion, perhaps moving with variable speed, up to some Markov time. More precisely, there is a standard Brownian motion $W_{t}^{f}=W_{t}=S_{t}+i V_{t}, 0<t<\infty$, such that $W_{0}=F(0)$, and a strictly increasing continuous random function $\lambda_{f}(t)=\lambda(t), 0<t<\tau_{D}$, such that $F\left(Z_{t}\right)=W_{\lambda(t)}$, $0<t<\tau_{D}$. The time $\lambda\left(\tau_{D}\right)$ is a Markov time for $W_{t}$, and will be shortened to $\gamma_{f}=\gamma$. See [7] for more detail about Lévy's theorem. Since $Z_{\tau_{D}}$ is uniformly distributed under $P$, for any real number $\alpha$ we have

$$
m\{f>\alpha\}=P\left(f\left(Z_{\tau_{D}}\right)>\alpha\right)=P\left(S_{\gamma}>\alpha\right) .
$$

The corresponding result holds for $\tilde{f}$, and, more generally, if $A$ is any Borel subset of $\mathbf{C}$,

$$
m\left\{e^{i \theta}: F\left(e^{i \theta}\right) \in A\right\}=P\left(W_{\gamma} \in A\right) .
$$

These equations imply $\|\tilde{f}\|_{p}^{p}=E\left|V_{\gamma}\right|^{p}$ and $\|f\|_{H^{p}}^{p}=E\left|W_{\gamma}\right|^{p}$. Now let $s_{i}, 1<i<n$, and $p_{i}, 1<i<n$, be as before. If $g$ is a real valued function on $T$ such that 
$m\left\{g=s_{i}\right\}=p_{i}$, then $G(0)=0$, and

$$
m\left(S_{\gamma_{g}}^{g}=s_{i}\right)=p_{i},
$$

so that, by Theorem 4.1, with $W_{t}^{g}$ in place of $Z_{t}$, if

$$
\eta_{g}=\inf \left\{t: W_{t}^{g} \notin R(s, p)\right\} \text {, }
$$

we have

$$
E\left|V_{\eta_{z}}^{g}\right|^{\rho}<E\left|V_{\gamma_{z}}^{g}\right|^{\rho}=\|\tilde{g}\|_{\rho}^{\rho}, \quad 1<\rho<2 .
$$

Now $E\left|V_{\eta_{z}}^{g}\right|^{\rho}$ does not depend on $g$. Since $W_{l}^{g}$ is standard Brownian motion started at 0 ,

$$
E\left|V_{\eta_{z}}^{g}\right|^{\rho}=E\left|Y_{\tau_{R(s, p)}}\right|^{\rho} .
$$

Thus, if a rearrangement $h$ of $g$ could be found such that $\eta_{h}=\gamma_{h}$, we would have

$$
\|\tilde{h}\|_{\rho}<\|\tilde{g}\|_{\rho}, \quad 1<\rho<2
$$

so $h$ would be extremal in the sense of (1.4).

There is such a rearrangement. If $H(z)$ is a universal covering map of $D$ onto $R(s, p)$ (see [1] for a discussion of such maps), then $f(D) \subset R(s, p)$ and it is impossible that $\lim H(z)=z_{0} \in R(s, p)$ if $z \in D$ moves continuously to $T$. This, and the fact that limit $_{t \rightarrow \tau_{D}} H\left(Z_{t}\right)$ exists (see [7]) implies that $\tau_{D}=\inf \{t>0$ : $\left.H\left(Z_{t}\right) \notin R(s, p)\right\}$, so that $\gamma_{h}=\eta_{h}$. This completes the proof of the existence part of Theorem 1.2 for the conjugate $L^{p}$ norms, $1<p<2$, in the case $\int_{T} f d m=0$, a restriction easily removed.

Next the nonexistence part of Theorem 1.1 for the conjugate $L^{p}$ norms for these same exponents will be proved. Let $q_{i}, 1<i<n$, and $s_{i}, 1<i<n$, be as before and assume $0<\Sigma q_{i}<1$. Let the function $k$ on $T$ satisfy $m\left\{k=s_{i}\right\}=q_{i}, 1<i<$ $n$, and $m\{k=0\}=1-\Sigma q_{i}$. Just as in the proof of (4.5)', Theorem 4.1 implies

$$
E\left|Y_{T(s, q)}\right|^{p}<\|\tilde{k}\|_{p}^{p}, \quad 1<p<2 .
$$

It cannot happen that $P\left(\gamma_{k}=0\right)>0$, for this would imply

$$
P\left(K\left(Z_{t}\right)=K(0)=0,0<t<\tau_{D}\right)>0,
$$

so that $K$ would vanish on a continuous (Brownian) path from 0 to $T$ and thus vanish everywhere. Thus Theorem 4.2 implies

$$
E\left|Y_{T(s, q)}\right|^{p}<\|\tilde{k}\|_{p}^{p}, \quad 1<p<2,
$$

and of course (4.14) holds if $k$ is replaced by any rearrangement of $k$, so that the argument showing nonexistence of a rearrangement of $k$ minimizing the conjugate $L^{p}$ norm can be completed by showing there are rearrangements $j$ of $k$ with $\|\tilde{j}\|_{p}^{p}$ arbitrarily close to $E\left|Y_{T(s, q)}\right|^{p}$.

Let $\varepsilon>0$ and let $R(s, p, \varepsilon)$ be the region $R(s, p)-\{i y: \delta<y<\varepsilon\}$, where $\delta>0$ is chosen so that $P\left(X_{\tau_{R(s, 0)}}=0\right)=1-\Sigma q_{i}$. Such a choice of $\delta$ is always possible since $P\left(Z_{t} \in\{i y: 0<y<\varepsilon\}\right.$ for some $\left.t<s\right)=1$ for each $s>0$. Now

$$
\begin{aligned}
& E\left|Y_{\tau_{R(s, p)}}\right|^{p}-E\left|Y_{\tau_{R(s, p, e)}}\right|^{p}=E\left(E_{Z_{\tau_{R(s, p, e)}}}\left|Y_{\tau_{R(s, p)}}\right|^{p}-\left|Y_{\tau_{R(s, p, e)}}\right|^{p}\right) I\left(X_{\tau_{R(s, p, e)}}=0\right) \\
& \rightarrow E\left|Y_{\tau_{R(s, p)}}\right|^{p}\left(1-\sum q_{i}\right) \text { as } \varepsilon \rightarrow 0,
\end{aligned}
$$


due to the continuity of the harmonic function $E_{z}\left|Y_{\tau_{R(s, p)}}\right|^{p}$. Thus, if $1<p<2$,

$$
E\left|Y_{\tau_{R(s, p, e)}}\right|^{p} \rightarrow E\left|Y_{T(s, q)}\right|^{p} \quad \text { as } \varepsilon \rightarrow 0 .
$$

Similarly

$$
P\left(X_{\tau_{R(s, p, e)}}=s_{i}\right) \rightarrow P\left(X_{T(s, q)}=s_{i}\right)=q_{i} \quad \text { as } \varepsilon \rightarrow 0 .
$$

Let $N_{e}(z)$ be the universal covering map of $D$ onto $R(s, p, \varepsilon)$ which takes 0 to 0 . Then $\gamma_{n_{\varepsilon}}=\inf \left\{t: W_{T}^{n_{z}} \notin R(s, p, \varepsilon)\right\}$, so that (4.15) and (4.16) imply

$$
\begin{gathered}
m\left\{n_{\varepsilon}=s_{i}\right\} \rightarrow m\left\{k=s_{i}\right\} \quad \text { as } \varepsilon \rightarrow 0, \quad \text { and } \\
\left\|\tilde{n}_{\varepsilon}\right\|_{p}^{p} \rightarrow E\left|Y_{T(s, q)}\right|^{p} \quad \text { as } \varepsilon \rightarrow 0 .
\end{gathered}
$$

Even though $n_{e}$ does not have exactly the distribution of $k$, it is not hard to use these to establish the existence of functions $m_{e}$, slight alterations of $n_{e}$, such that $m_{e}$ and $k$ have the same distribution and $\left\|\tilde{m}_{e}\right\|_{p}^{p} \rightarrow E\left|Y_{T(s, q)}\right|^{p}$.

There is no question that, in the cases where Theorem 1.1 guarantees an extremal rearrangement exists, these rearrangements do not have a regular form, whenever $n>2$. They probably become very chaotic when $m\left\{f=a_{1}\right\}$ is large for some $a_{i}$ close to $\int_{T} f d m$. Some less discrete functions have an extremal rearrangement which is very regular, and which will now be described. If $g$ is a function on $T$ such that $m\{g=0\}=0$, let $g_{e}$ be the rearrangement of $g$ which satisfies $g_{e}\left(e^{i \theta}\right)=$ $g_{e}\left(e^{-i \theta}\right)$, is positive on $\left\{e^{i \theta}:-\alpha<\theta<\alpha\right\}$, negative on $\left\{e^{i \theta}: \alpha<\theta<2 \pi-\alpha\right\}$, is not decreasing as $\theta$ increases from 0 to $\alpha$ and not decreasing as $\theta$ increases from $\alpha$ to $\pi$. Here $\alpha$ is $m\{g>0\} \pi$. Now if $F$ is a univalent map from $D$ to a simply connected region $R$ which has a complement that can be written as a union of vertical line segments centered on the real axis (as $R(s, p)$ has), which is contained in some strip $\{x+i y$ : $-m<x<m\}$ for a finite number $m$ (as $R(s, p)$ is), and which maps 0 onto 0 , then it can be shown by a slight alteration of the proof of the existence part of Theorem 1.1 that $f$ is an extremal rearrangement of itself in all the senses of (1.4), (1.5), (1.6), and (1.7). Furthermore if $F$ has a continuous extension from $\bar{D}$ to $\bar{R}$, except for two boundary points where $\operatorname{Re} F\left(e^{i \theta}\right)$ changes from positive to negative, and if $F(1)$ is real and positive, it is not hard to show that $f=f_{e}$. There are many examples of such functions. We cannot characterize those distributions for $f$ for which $f_{e}$ is extremal, without recourse to analytic functions. Note that it is quite easy to show that $f_{e}$ is in $\operatorname{Re} H^{1}$ if and only if $f_{d}$ in $\operatorname{Re} H^{1}$, since the two jumps of $f_{e}$ resemble the one jump of $f_{d}$. This fact could be used to prove much of what was proved in $\$ 2$, in the special case that $f_{e}$ is extremal.

5. An example. In this section it will be shown that if $p$ is a real number in $(0,1)$ there is a bounded real valued function $f_{p}=f$ on $T$ such that

$$
\|\tilde{f}\|_{p}>\left\|\tilde{f_{s}}\right\|_{p} \text {. }
$$

The exponent $p \in(0,1)$ is to be considered as fixed, and let $\Gamma$ be the strip $\{x+i y$ : $-1<x<1\}$. For $x= \pm 1$ and $-\infty<y<\infty$, let $\gamma(x+i y)=\varphi(y)$ be harmonic density with respect to the region $\Gamma$ and the point 0 , meaning

$$
h(0)=\int_{-\infty}^{\infty} h(1+i y) \varphi(y) d y+\int_{-\infty}^{\infty} h(-1+i y) \varphi(y) d y,
$$


whenever $h$ is harmonic and bounded on $\Gamma$ and continuous on $\bar{\Gamma}$. It is not hard to calculate $\varphi$, but for our purposes it is enough to know that $\varphi$ is symmetric about 0 , and that $\varphi(y)<K e^{-\alpha|y|}$ for all $y$ and some positive constants $K$ and $\alpha$. Define

$$
\delta(t)=|t|^{p}-2 \int_{-\infty}^{\infty}|s|^{p} \varphi(t-s) d s, \quad-\infty<t<\infty
$$

The term subtracted in this expression is the canonical harmonic function with boundary values $|y|^{p}$, evaluated at the point it. Since $\int_{-\infty}^{\infty} \varphi(y) d y=1$, and since $\varphi$ is symmetric about 0 , we have

$$
\begin{aligned}
\delta(t)= & 2 \int_{-\infty}^{\infty}\left(|t|^{p}-|s|^{p}\right) \varphi(t-s) d s \\
= & 2 \int_{0}^{t}\left[2|t|^{p}-|t-s|^{p}-|t+s|^{p}\right] \varphi(s) d s \\
& +2 \int_{\{s:|t-s|>t\}}\left(|t|^{p}-|s|^{p}\right) \varphi(t-s) d s \\
= & 2 \int_{0}^{t}\left[\int_{t-s}^{t+s} p(p-1)|x|^{p-2} d x\right] \varphi(s) d s \\
& +2 \int_{\{|t-s|>t\}}\left(|t|^{p}-|s|^{p}\right) \varphi(t-s) d s \\
= & A(t)+B(t) .
\end{aligned}
$$

Since $\varphi(y)<K e^{-\alpha|y|}$, it is easy to show that $B(t)=O\left(e^{-\beta|t|}\right)$, as $|t| \rightarrow \infty$, for some positive constant $\beta$, and, since $p(p-1)$ is negative, there exist positive numbers $\eta$ and $\nu$ such that

$$
A(t)<-\eta|t|^{p-2} \text { if }|t|>\nu .
$$

Thus $\delta(t)$ is negative for all large enough $t$, and since both $A(t)$ and $B(t)$ approach 0 as $|t| \rightarrow \infty$, we have $\delta(t) \rightarrow 0$ as $|t| \rightarrow \infty$. Let

$$
m=\min \{\delta(t):-\infty<t<\infty\},
$$

and let $\eta$ satisfy $\delta(\eta)=m$.

Let $Z_{t}$ be as in the last section. If $\tau$ is any stopping time for $Z_{t}$ such that $\tau<\tau_{\Gamma}$ and $P\left(X_{\tau}=0\right)+P\left(X_{\tau}=1\right)+P\left(X_{\tau}=-1\right)=1$, we have

$$
\begin{aligned}
E\left|Y_{\tau}\right|^{p} & =E\left|Y_{\tau_{\Gamma}}\right|^{p}-E\left(\left|Y_{\tau_{\Gamma}}^{p}\right|-\left|Y_{\tau}\right|^{p}\right) I\left(\tau<\tau_{\Gamma}\right) \\
& =E\left|Y_{\tau_{\Gamma}}\right|^{p}-E E_{Z_{\Gamma}}\left(\left|Y_{\tau_{\Gamma}}\right|^{p}-\left|Y_{\tau}\right|^{p}\right) I\left(X_{\tau}=0\right) \\
& =E\left|Y_{\tau_{\Gamma}}\right|^{p}+E \delta\left(Y_{\tau}\right) I\left(X_{\tau}=0\right) .
\end{aligned}
$$

Now for $0<\varepsilon<1$, let $R(\varepsilon)=\Gamma-\{i y: \eta-a<y<\eta+a\}$ and

$$
S(\varepsilon)=\Gamma-\{i y:|y|>b\},
$$

where $a=a(\varepsilon)$ and $b=b(\varepsilon)$ are the unique positive numbers such that

$$
P\left(X_{\tau_{R(e)}}=0\right)=\varepsilon \text { and } P\left(X_{\tau_{S(e)}}=0\right)=\varepsilon .
$$

Since $b(\varepsilon) \rightarrow 0$ as $\varepsilon \rightarrow 0$, (5.1) implies

$$
\lim _{\varepsilon \rightarrow 0} \varepsilon^{-1}\left[E\left|Y_{\tau_{R(e)}}\right|^{p}-E\left|Y_{\tau_{\mathbf{r}}}\right|^{p}\right]=-m>0,
$$


and, since $\delta$ vanishes at infinity, and $a(\varepsilon) \rightarrow \infty$ as $\varepsilon \rightarrow 0$,

$$
\lim _{\varepsilon \rightarrow 0} \varepsilon^{-1}\left[\left|Y_{\tau_{\text {S(e) }} \mid}\right|^{p}-E\left|Y_{\tau_{\mathrm{r}}}\right|^{p}\right]=0
$$

Thus there is a positive number, which will be denoted $\lambda$, such that

$$
E\left|Y_{\tau_{R(\lambda)}}\right|^{p}>E\left|Y_{\tau_{s(\lambda)}}\right|^{p} \text {. }
$$

Now Baernstein has shown in [2] that, if $h=h_{s}$, and $m\{h=1\}=m\{h=-1\}=$ $(1-\lambda) / 2$ and $m\{h=0\}=\lambda$, then $H_{s}$ maps $D$ univalently onto $S(\delta)$ and maps 0 onto 0 , and arguments like those of the last section give

$$
E\left|Y_{\tau_{s(\lambda)}}\right|^{p}=\left\|\tilde{h_{s}}\right\|_{p}^{p}
$$

If $G$ is a universal covering map of $D$ onto $R(\lambda)$ which takes 0 onto 0 , then again arguing as in the last section, we have

$$
m\{g=1\}=m\{g=-1\}=(1-\lambda) / 2, \quad m\{g=0\}=\lambda,
$$

and

$$
E\left|Y_{\tau_{R(\lambda)}}\right|^{p}=\|\tilde{g}\|_{p}^{p}
$$

Since $g$ and $h$ have the same distribution, $g_{s}=h_{s}$. Thus (5.2), (5.3), and (5.4) imply

$$
\|\tilde{g}\|_{p}^{p}>\left\|\tilde{g}_{s}\right\|_{p}^{p}
$$

\section{REFERENCES}

1. L. Alfhors, Conformal invariants, McGraw-Hill, New York, 1973.

2. A. Baernstein, Some sharp inequalities for conjugate functions, Indiana Univ. Math. J. 27 (1978), 833-852.

3. __ Some sharp inequalities for conjugate functions, Proc. Sympos. Pure Math., vol. 35, part 1, Amer. Math. Soc., Providence, R. I., 1979, pp. 409-416.

4. D. L. Burkholder, R. F. Gundy and M. L. Silverstein, A maximal function characterization of the class $H^{p}$, Trans. Amer. Math. Soc. 157 (1971), 137-153.

5. D. L. Burkholder and R. F. Gundy, Boundary behavior of harmonic functions in a half space and Brownian motion, Ann. Inst. Fourier (Grenoble) 23 (1972), 195-212.

6. R. R. Coifman and G. Weiss, Extensions of Hardy spaces and their uses in analysis, Bull. Amer. Math. Soc. 83 (1977), 569-645.

7. B. Davis, Brownian motion and analytic functions, Ann. Probability 7 (1979), 913-932.

8. M. Essen and D. Shea (to appear).

9. A. Garsia, Martingale inequalities, Benjamin, New York, 1973.

10. C. Herz, $H_{p}$ spaces of martingales, $0<p<1$, Z. Wahrscheinlichkeitstheorie und Verw. Gebiete 28 (1974), 189-205.

11. G. A. Hunt, Some theorems concerning Brownian motion, Trans. Amer. Math. Soc. 81 (1956), 294-319.

12. A. Zygmund, Trigonometric series, Cambridge Univ. Press, Cambridge, 1968.

Department of Statistics, Purdue University, West Lafayette, Indiana 47907 